\title{
Low-rank matrix estimation-based spatio-temporal image reconstruction for dynamic photoacoustic computed tomography
}

Kun Wang, Jun Xia, Changhui Li, Lihong V. Wang, Mark A. Anastasio

Kun Wang, Jun Xia, Changhui Li, Lihong V. Wang, Mark A. Anastasio, "Lowrank matrix estimation-based spatio-temporal image reconstruction for dynamic photoacoustic computed tomography," Proc. SPIE 8943, Photons Plus Ultrasound: Imaging and Sensing 2014, 89432I (3 March 2014); doi: $10.1117 / 12.2041850$ 


\title{
Low-rank matrix estimation-based spatio-temporal image reconstruction for dynamic photoacoustic computed tomography
}

\author{
Kun Wang ${ }^{a}$, Jun Xia ${ }^{a}$, Changhui $\mathrm{Li}^{b}$, Lihong V. Wang ${ }^{a}$, and Mark A. Anastasio ${ }^{a}$ \\ ${ }^{a}$ Department of Biomedical Engineering, Washington University in St. Louis, \\ St. Louis, MO 63130 \\ ${ }^{b}$ Department of Biomedical Engineering, Peking University, Beijing, 100871 P.R.China
}

\begin{abstract}
In order to monitor dynamic physiological events in near-real time, a variety of photoacoustic computed tomography (PACT) systems have been developed that can rapidly acquire data. Previously reported studies of dynamic PACT have employed conventional static methods to reconstruct a temporally ordered sequence of images on a frame-by-frame basis. Frame-by-frame image reconstruction (FBFIR) methods fail to exploit correlations between data frames and are known to be statistically and computationally suboptimal. In this study, a low-rank matrix estimation-based spatio-temporal image reconstruction (LRME-STIR) method is investigated for dynamic PACT applications. The LRME-STIR method is based on the observation that, in many PACT applications, the number of frames is much greater than the rank of the ideal noiseless data matrix. Using computer-simulated photoacoustic data, the performance of the LRME-STIR method is compared with that of conventional FBFIR method. The results demonstrate that LRME-STIR method is not only computationally more efficient but also produces more accurate dynamic PACT images than a conventional FBFIR method.
\end{abstract}

Keywords: photoacoustic computed tomography; optoacoustic tomography; dynamic imaging; low-rank matrix estimation

\section{INTRODUCTION}

Photoacoustic computed tomography (PACT), also known as optoacoustic tomography, is an emerging imaging modality that has great potential for a wide range of biomedical imaging applications. ${ }^{1-3}$ PACT is a hybrid imaging modality that combines the high spatial resolution of ultrasound imaging and the high soft tissue contrast of optical imaging. ${ }^{4-6}$ In PACT, a short laser pulse is employed to illuminate an object and internal acoustic wavefields are produced via the thermoacoustic effect. The acoustic wavefields propagate out of the object and are measured by use of ultrasonic transducers. ${ }^{4,5,7}$ From the measured wavefield data, an image that depicts the absorbed optical energy density distribution within the object, hereafter referred to as the object function, is produced by use of a reconstruction algorithm. The vast majority of PACT image reconstruction algorithms developed to date assume static imaging conditions, in which the sought-after object function is independent of time.

In PACT studies that involve dynamic physiological processes, the object function changes with time. The goal of dynamic $\mathrm{PACT}^{8-11}$ is to reconstruct a sequence of object function estimates that correspond to a collection of time points. These temporal samples of the object function will be referred to as 'object frames'. At each temporal sample in a dynamic PACT study, a static PACT data set is recorded. This data set will be referred to as a 'data frame'. It is assumed that the object function remains static during acquisition of each data frame, which can be approximately satisfied if the temporal resolution of the imaging system is sufficiently high. An estimate of each object frame can be reconstructed from the corresponding data frame by use of a conventional

(Send correspondence to:)

Kun Wang: E-mail: kunwang.kw@gmail.com,

Mark Anastasio: E-mail: anastasio@wustl.edu

Photons Plus Ultrasound: Imaging and Sensing 2014, edited by Alexander A. Oraevsky, Lihong V. Wang, Proc. of SPIE Vol. 8943, 89432I - (C) 2014 SPIE - CCC code: 1605-7422/14/\$18 - doi: 10.1117/12.2041850 
static PACT reconstruction algorithm. This image reconstruction approach will be referred to as a frame-byframe image reconstruction (FBFIR) method, and has been utilized in the vast majority of previous studies of dynamic PACT. ${ }^{9-12}$ A limitation of FBFIR methods is that they fail to exploit correlations between data frames and are therefore known to be statistically and computationally suboptimal ${ }^{13-15}$.

Several image reconstruction methods have been proposed for dynamic PACT. ${ }^{9-12}$ Gamelin et. al proposed to synthesize a densely sampled data frame by estimating the pressure data at the locations between transducers. ${ }^{16}$ It was demonstrated that use of the method could improve the temporal resolution of a PACT imaging system by permitting sufficiently accurate image reconstruction from data frames of reduced sizes. However, being an FBFIR method, it is subject to the limitations discussed above. In a different study, an image-domain KarhunenLoève (KL) filter (i.e, principal component filter) and an independent component analysis filter were applied to the images reconstructed by use of an FBFIR method. ${ }^{17}$

Unlike FBFIR methods, spatio-temporal image reconstruction (STIR) methods for dynamic tomography modalities jointly reconstruct the sequence of object frame estimates by use of all of the data frames. These algorithms exploit statistical correlations or deterministic linear dependency between either data or image frames. ${ }^{18,19}$ In this way, STIR methods can circumvent the limitations of FBFIR methods that are described above. A variety of advanced STIR methods have been developed for use with established dynamic tomography modalities. ${ }^{13,20-22}$ When utilized in conjunction with optimized data-acquisition protocols, STIR methods can improve the temporal resolution of an imaging system, so that rapid dynamic physiological events can be visualized. ${ }^{15,20}$ Despite these advantages, the development and investigation of STIR methods for dynamic PACT remains largely unexplored.

In this study, a low-rank matrix estimation-based spatio-temporal image reconstruction (LRME-STIR) method is investigated for dynamic PACT applications. The LRME-STIR method is based on the observation that, in many PACT applications, the number of frames is much greater than the rank of the ideal noiseless data matrix. Our work is inspired by the successful application of similar ideas in dynamic magnetic resonance imaging. ${ }^{15,21}$ The remainder of the article is organized as follows. The static and dynamic PACT image reconstruction problems are reviewed in Section 2. In Section 3, we describe the LRME-STIR method. Computer simulation studies and results are provided in Section 4 and 5 respectively. We conclude with a brief summary in Section 6.

\section{BACKGROUND}

\subsection{Static image reconstruction in conventional PACT}

A static PACT imaging system can be accurately described by a continuous-to-discrete (C-D) imaging model $\operatorname{as}^{5,7,23-25}$

$$
[\mathbf{g}]_{j I+i}=\left.h^{e}(t) *_{t} \frac{1}{\Omega_{j}} \int_{\Omega_{j}} d \mathbf{r}^{\prime} \frac{\beta}{4 \pi C_{p}} \int_{\mathcal{V}} d \mathbf{r} A^{\mathrm{s}}(\mathbf{r}) \frac{d}{d t} \frac{\delta\left(t-\frac{\left|\mathbf{r}^{\prime}-\mathbf{r}\right|}{c_{0}}\right)}{\left|\mathbf{r}^{\prime}-\mathbf{r}\right|}\right|_{t=i \Delta T}, \quad \underset{j=0,1, \cdots, J-1}{i=0, \ldots, I-1},
$$

where $h^{e}(t)$ is the electrical impulse response (EIR) of the transducer, ${ }^{24,26} *_{t}$ denotes the temporal convolution operation, $\delta(t)$ is the one-dimensional Dirac delta function, and $\beta, c_{0}$ and $C_{p}$ denote the thermal coefficient of volume expansion, the (constant) speed-of-sound, and the specific heat capacity of the medium at constant pressure, respectively. The static object function, $A^{\mathrm{s}}(\mathbf{r})$, is assumed to be bounded and contained within the volume $\mathcal{V}$. The vector $\mathbf{g} \in \mathbb{R}^{I J}$ represents a lexicographically ordered collection of sampled values of the electrical signals produced by the ultrasonic transducers, where $J$ and $I$ denote the number of transducers employed in the imaging system and the number of temporal samples recorded by each transducer, respectively. The notation $[\mathbf{g}]_{j I+i}$ will be utilized to denote the $(j I+i)$-th element of $\mathbf{g}$. Here, the integer-valued indices $j$ and $i$ describe the transducer location and temporal sample, respectively. The quantity $\Omega_{j}$ denotes the detection area of the $j$-th transducer and $\Delta_{t}$ the temporal sampling interval. By introducing an operator $\mathcal{H}$ to denote the C-D mapping from $A^{\mathrm{s}}(\mathbf{r})$ to $\mathbf{g}$, Eqn. (1) can be written as

$$
\mathrm{g}=\mathcal{H} A^{\mathrm{s}},
$$

where $\mathcal{H}$ will be referred to as a static PACT imaging operator.

Based on Eqn. (1), iterative image reconstruction algorithms have been developed for estimation of $A^{\mathrm{s}}(\mathbf{r}){ }^{24,25,27}$ When the transducer size is small and/or the object is located near the center of a relatively large measurement 
geometry, the surface integral over $\Omega_{j}$ can be neglected. In these cases, a variety of analytic formulae ${ }^{28-31}$ can also be employed for image reconstruction after de-convolving the EIR from the recorded signals. ${ }^{32}$ Linear static image reconstruction algorithms will be described as

$$
\hat{\mathbf{A}}^{\mathrm{s}}=\mathbf{B g},
$$

where the matrix $\mathbf{B} \in \mathbb{R}^{N \times I J}$ represents a discrete image reconstruction operator and $\hat{\mathbf{A}}^{\mathrm{s}} \in \mathbb{R}^{N}$ is the reconstructed digital image arranged in a lexicographical order with $N$ being the number of pixels or voxels.

\subsection{Dynamic PACT and frame-by-frame image reconstruction}

In dynamic PACT, the optical absorption coefficient $\mu_{a}\left(\mathbf{r}, t^{\mathrm{s}}\right)$ and the optical fluence $\Phi\left(\mathbf{r}, t^{\mathrm{s}}\right)$ are time-dependent. ${ }^{4}$ The time-dependent object function is accordingly defined as $A\left(\mathbf{r}, t^{\mathrm{s}}\right) \equiv \Phi\left(\mathbf{r}, t^{\mathrm{s}}\right) \mu_{a}\left(\mathbf{r}, t^{\mathrm{s}}\right)$. Here and throughout the manuscript, $t^{\mathrm{s}}$ (or the corresponding index $k$ defined below) will be referred to as a slow-time (i.e., the time of each frame) coordinate, while the time coordinate $t$ (or the corresponding index $i$ ) in Eqn. (1) will be referred to as a fast-time (i.e., the arrival time of acoustic signals) coordinate.

The $k$-th frame of the dynamic object is defined as

$$
\left.A_{k}(\mathbf{r}) \equiv \Phi\left(\mathbf{r}, t^{\mathrm{s}}\right) \mu_{a}\left(\mathbf{r}, t^{\mathrm{s}}\right)\right|_{t^{\mathrm{s}}=k \Delta_{t^{\mathrm{s}}}}, \quad \text { for } \quad k=0,1, \cdots, K-1,
$$

where $K$ denotes the number of temporal samples, indexed by $k$, with temporal sampling interval $\Delta_{t^{s}}$. Replacing $A^{\mathrm{s}}(\mathbf{r})$ in Eqn. (2) by $A_{k}(\mathbf{r})$ yields

$$
\mathbf{g}_{k}=\mathcal{H} A_{k},
$$

where $\mathbf{g}_{k} \in \mathbb{R}^{I J}$ denotes the $k$-th data frame.

The goal of dynamic PACT is to estimate the collection of object frames $\left\{A_{k}(\mathbf{r})\right\}_{k=0}^{K-1}$ from the measured data frames of $\left\{\mathbf{g}_{k}\right\}_{k=0}^{K-1}$. To accomplish this, a linear frame-by-frame image reconstruction (FBFIR) method operates as

$$
\hat{\mathbf{A}}_{k}=\mathbf{B g}_{k}, \quad \text { for } \quad k=0,1, \cdots, K-1,
$$

where $\hat{\mathbf{A}}_{k} \in \mathbb{R}^{N}$ represents a finite-dimensional estimate of the $k$-th object frame $A_{k}(\mathbf{r})$. Note that $\mathbf{B}$ has to be applied $K$ times to obtain the sought-after sequence of image estimates $\left\{\hat{\mathbf{A}}_{k}\right\}_{k=0}^{K-1}$. We define the matrices $\hat{\mathbf{A}} \in \mathbb{R}^{N \times K}$ and $\mathbf{G} \in \mathbb{R}^{I J \times K}$ as

$$
\hat{\mathbf{A}}=\left[\hat{\mathbf{A}}_{0}\left|\hat{\mathbf{A}}_{1}\right| \cdots \mid \hat{\mathbf{A}}_{K-1}\right], \quad \text { and } \quad \mathbf{G}=\left[\mathbf{g}_{0}\left|\mathbf{g}_{1}\right| \cdots \mid \mathbf{g}_{K-1}\right],
$$

where $\mathbf{G}$ will be referred to as the data matrix. In terms of these matrices, Eqn. (6) can be expressed as

$$
\hat{\mathbf{A}}=\mathbf{B G} \text {. }
$$

\section{LOW-RANK MATRIX ESTIMATION-BASED STIR FOR DYNAMIC PACT}

\subsection{Spatio-temporal image reconstruction method}

Consider the singular-value decomposition $(\mathrm{SVD})^{18}$ of the data matrix $\mathbf{G}$ :

$$
\mathbf{G}=\sum_{k=0}^{R-1} \mu_{k} \mathbf{v}_{k} \mathbf{u}_{k}^{\dagger},
$$

where $R$ is the rank of $\mathbf{G}$, the superscript $\dagger$ denotes the matrix adjoint, and $\left\{\mathbf{u}_{k}, \mathbf{v}_{k}, \mu_{k}^{2}\right\}_{k=0}^{R-1}$ is the associated singular system. Note that the SVD of the data matrix can be calculated efficiently because for many dynamic PACT applications, the value of $K$ is on the order of $100 .{ }^{9-11}$ On substitution from Eqn. (9) into Eqn. (8), one obtains

$$
\hat{\mathbf{A}}=\sum_{k=0}^{R-1} \mu_{k} \mathbf{B v}_{k} \mathbf{u}_{k}^{\dagger}
$$

Equation (10) describes a STIR method that is mathematically equivalent to the FBFIR method in Eqns. (6) or (8). However, when the rank of the data matrix is far smaller than the number of slow-time frames, the number of applications of the static image reconstruction operator $\mathbf{B}$ will be reduced from $K$ to $R$, which will significantly reduce the computational burden. 


\subsection{Low-rank matrix estimation-based STIR method}

The derivation of the STIR formula in Eqn. (10) did not consider data noise. Because of measurement noise, the measured data matrix, denoted by $\underline{\mathbf{G}}$, is likely to be of full rank, i.e., $\underline{R}=K$, where $\underline{R}$ is the rank of $\underline{\mathbf{G}}$. Simply replacing $\mathbf{G}$ in Eqn. (10) with a full-rank data matrix $\underline{\mathbf{G}}$ will mitigate the computational advantages of the STIR method. Furthermore, directly applying the STIR formula with noisy measurements may result in image artifacts, just as would occur in FBFIR. These artifacts can be mitigated by incorporating regularization in the static image reconstruction operator $\mathbf{B} .^{25,27}$ Alternatively, an explicit denoising approach can be employed to estimate the noise-free $\mathbf{G}$, from which $\mathbf{A}$ can be reconstructed by use of algorithms developed for idealized noise-free measurements ${ }^{32}$ such as Eqn. (10). In this study, the latter strategy is employed.

Hereafter, random quantities are underlined. The measured data matrix $\underline{\mathbf{G}}$ can be expressed as

$$
\underline{\mathbf{G}}=\mathbf{G}+\underline{\mathbf{N}}
$$

where $\underline{\mathbf{N}}$ is a random noise matrix of dimensions $I J \times K$ and $\mathbf{G}$ is the noiseless data matrix. Estimation of $\mathbf{G}$ from $\underline{\mathbf{G}}$ is a classic image denoising problem. A variety of image denoising algorithms can be employed for this task, including recently studied sparsity-regularized denoising methods. ${ }^{32-35}$ In this study, denoising of $\underline{\mathbf{G}}$ was performed by solving the following optimization problem: ${ }^{15,36,37}$

$$
\hat{\mathbf{G}}=\arg \min _{\mathbf{G}} \frac{1}{2}\|\underline{\mathbf{G}}-\mathbf{G}\|_{\mathrm{F}}^{2}+\beta \operatorname{Rank}(\mathbf{G}),
$$

where $\operatorname{Rank}(\mathbf{G})$ denotes the $\operatorname{rank}$ of $\mathbf{G}, \beta$ is a regularization parameter, and $\|\cdot\|_{F}$ denotes the Frobenius norm. The Frobenius norm is defined as the square root of the sum of the absolute squares of the matrix's elements, and can be viewed as an extension of the $\ell_{2}$ vector norm to matrices. The penalty term $\operatorname{Rank}(\mathbf{G})$ will promote solutions $\hat{\mathbf{G}}$ that are of low rank. Equation (12), which will be referred to as the low-rank matrix estimation (LRME) problem, possesses a closed-form solution that can be calculated via the singular value hard thresholding (SVHT) estimator as ${ }^{38}$

$$
\hat{\mathbf{G}}=\sum_{k=0}^{\hat{R}-1} \underline{\mu}_{k} \underline{\mathbf{v}}_{k} \underline{\mathbf{u}}_{k}^{\dagger},
$$

where $\left\{\underline{\mathbf{u}}_{k}, \underline{\mathbf{v}}_{k}, \underline{\mu}_{k}^{2}\right\}$ denotes the singular system of $\underline{\mathbf{G}}$ and $\hat{R}$ is the maximum of $\left\{k \mid \underline{\mu} \underline{\mu}_{k}>\beta\right\}$.

Because both are conducted in the singular system of data matrices, the low-rank regularized data matrix denoising and the STIR method can be naturally combined as a two-step LRME-based STIR (LRME-STIR) method. Since we employed the SVHT estimator in Eqn. (13), we obtained not only a low-rank estimate $\hat{\mathbf{G}}$ but also its singular system as $\left\{\underline{\mathbf{u}}_{k}, \underline{\mathbf{v}}_{k}, \underline{\mu}_{k}^{2}\right\}_{k=0}^{\hat{R}-1}$. Therefore, applying the STIR formula to $\hat{\mathbf{G}}$ can be immediately obatined by replacing $R, \mu_{k}, \mathbf{v}_{k}$, and $\mathbf{u}_{k}$ in Eqn. (10) with $\hat{R}, \underline{\mu}_{k}, \underline{\mathbf{v}}_{k}$, and $\underline{\mathbf{u}}_{k}$, respectively. When $\hat{R} \ll K$, the LRME-STIR method can be computationally efficient.

\section{DESCRIPTIONS OF NUMERICAL STUDIES}

Computer-simulation studies were conducted to demonstrate the use of the STIR method for dynamic PACT reconstruction.

A schematic of the measurement geometry is shown in Fig. 1. This measurement geometry emulates an existing small-animal imaging system. ${ }^{39}$ We employed a time-varying object function that was spatially supported in a square region of size $11 \times 11 \mathrm{~mm}^{2}$. The object function was discretized along the slow-time coordinate into $K=90$ object frames according to Eqn. (4). Around the object, $J=512$ ultrasonic transducers were uniformly distributed on a ring of radius $25 \mathrm{~mm}$. Ninety data frames were simulated corresponding to the 90 object frames respectively. Each data frame was analytically calculated by use of Eqn. (5) with with $I=650$ at the fast-time sampling rate of $40 \mathrm{MHz}$. Accordingly, the noise-free data matrix $\mathbf{G}$ had dimensions of $332,800 \times 90$. The noise matrix $\underline{\mathbf{N}}$ contained white Gaussian entries with variance specified as $20 \%$ of $\|\mathbf{G}\|_{\mathrm{F}}^{2} /(I J K)$. Summation of $\underline{\mathbf{N}}$ and $\mathbf{G}$ resulted in the noisy data matrix $\underline{\mathbf{G}}$ according to Eqn. (11). 


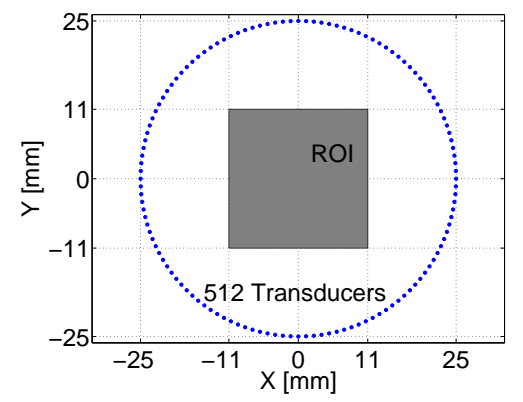

Figure 1. Schematic of the circular measurement geometry, where the shaded square represents a region-of-interest (ROI) and the blue dots represent 512 transducers uniformly distributed on a ring.

We implemented both the FBFIR and the LRME-STIR methods for imaging reconstruction. The linear static imaging operator B in both Eqns. (8) and (10) was defined as a discrete version of a two-dimensional FBP formula ${ }^{40}$ whose description in continuous form is given by:

$$
\hat{A}_{k}(\mathbf{r})=\frac{C_{p}}{\pi c_{0} \beta R^{\prime}} \int_{\Omega} d \mathbf{r}^{\prime} \int_{0}^{\frac{2 R^{\prime}}{c_{0}}} d t \frac{\partial}{\partial t} t \frac{\partial}{\partial t} \log \left|t^{2} c_{0}^{2}-\right| \mathbf{r}^{\prime}-\left.\mathbf{r}\right|^{2} \mid \int_{0}^{t} d \tau g_{k}\left(\mathbf{r}^{\prime}, \tau\right),
$$

where $R^{\prime}=25 \mathrm{~mm}$ was the scanning radius, $c_{0}=1.5 \mathrm{~mm} / \mu \mathrm{s}, \Omega$ was the measurement circle, $C_{p} / \beta$ was set to be 1.0 in arbitrary units (a.u), and the measured pressure $g_{k}\left(\mathbf{r}^{\prime}, t\right)$ for the $k$-th frame was a function of location $\mathbf{r}^{\prime} \in \Omega$ and fast-time $t$. Since we assumed idealized point-like transducers, samples of $g_{k}\left(\mathbf{r}^{\prime}, t\right)$ were equivalent to the simulated measurement data.

\section{NUMERICAL RESULTS}

Images reconstructed from noise-free data are shown in Fig. 2. Since the data contain no noise, we applied the STIR formula in Eqn. (10) directly to the noise-free data. As expected, images reconstructed by use of the STIR method (Fig. 2-(c)) are nearly identical to those reconstructed by use of the FBFIR method (Fig. 2-(b)). Both algorithms accurately reconstruct absorbed optical energy density distribution as specified by the phantom in Fig. 2-(a). This observation holds true for all 90 slow-time frames. Pixel time-activity curves corresponding to the STIR method agree with the phantom accurately as shown in Fig. 3. The results demonstrate that the STIR method is mathematically equivalent to the FBFIR method. However, the STIR method was 15 times faster than the FBFIR method because it required the application of the FBP algorithm 6 times in comparison to the 15 times required for the FBFIR method.
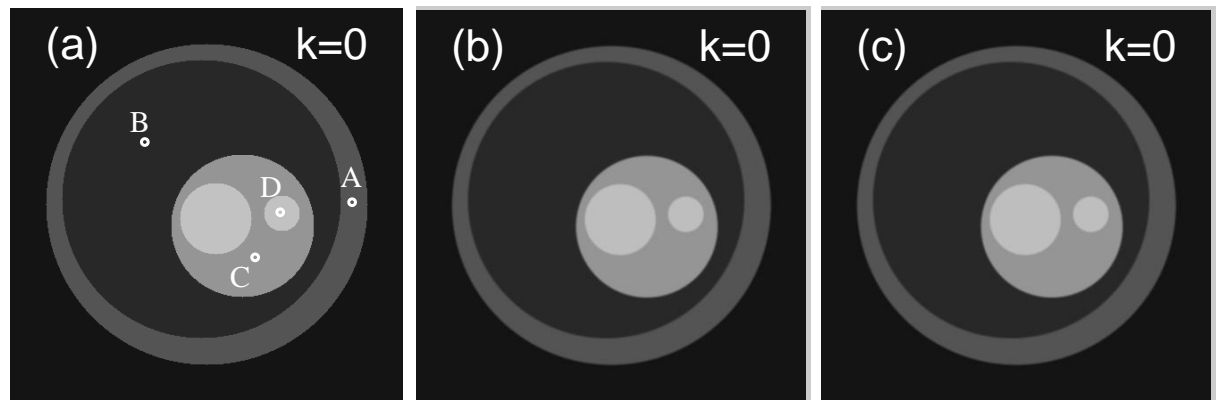

Figure 2. The 0-th frame of (a) the dynamic object, and the images reconstructed by use of (b) the FBFIR and (c) the STIR methods from the noise-free data, respectively.

Images reconstructed from noisy data are shown in Fig. 4. As expected, images reconstructed by use of the LRME-STIR method (Fig. 4-(b)) appear to have a reduced noise level in comparison to the images reconstructed 


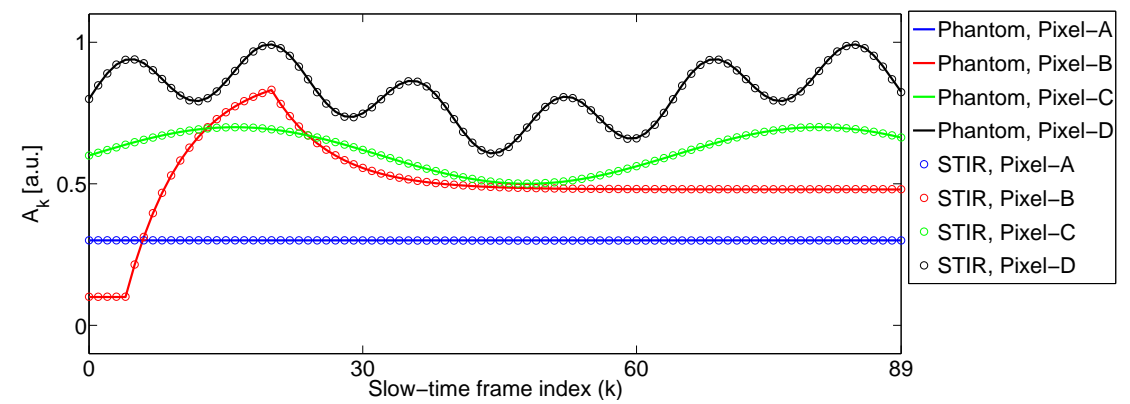

Figure 3. Pixel time-activity-curves of images reconstructed by use of the STIR method from the noise-free data.

by use of the FBFIR method (Fig. 4-(b)). The reduced noise level can be more evidently observed in Fig. 5, which displays the collection of time-activity curves of pixels on the line $y=0$. The image reconstructed by use of the FBFIR method contains multiple vertical streak-artifacts as shown in Fig. 5-(b). These steaks are caused by the additive random noise, which are not attended in the conventional FBFIR method. In contrast, the LRME-STIR method effectively mitigated these streaks as shown in Fig. 4-(c). This is because the LRME step reduced the noise level of the data matrix. After the LRME, the noise-reduced data matrix $\hat{\mathbf{G}}$ has a rank of 4. Accordingly, by use of the STIR formula, the computational time was reduced by a factor of 20 .
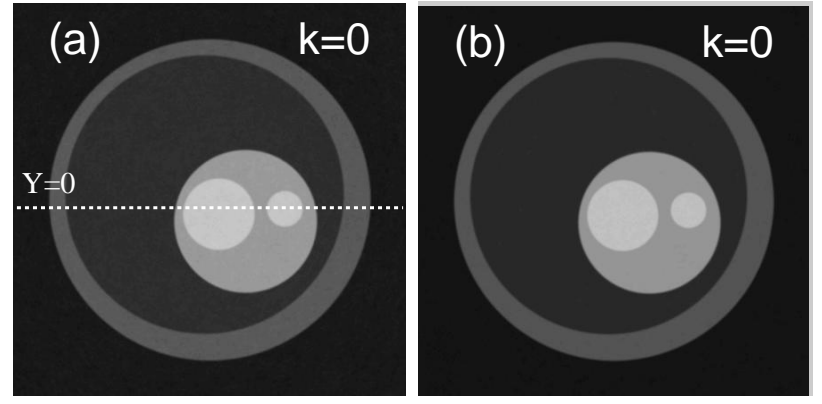

Figure 4. The 0-th frame of the images reconstructed by use of (a) the FBFIR and (b) the LRME-STIR methods from the noisy data.
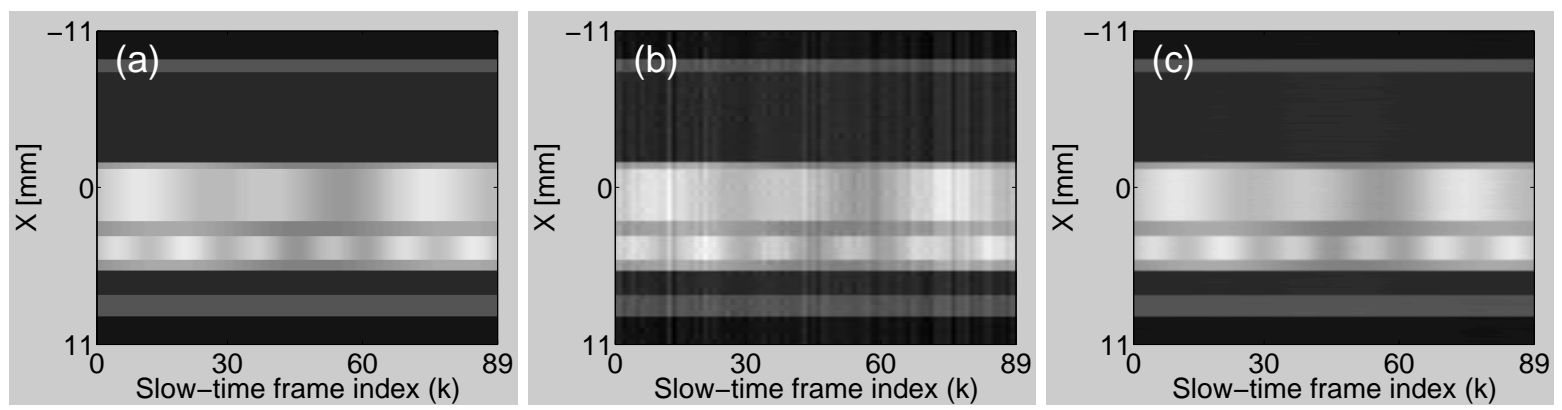

Figure 5. Estimates of $\left\{A_{k}(x, y=0)\right\}_{k=0}^{89}$ by use of (b) the FBFIR method and (c) the LRME-STIR method from the noisy data contaminated with $20 \%$ Gaussian white noise, respectively. The original phantom is plotted in panel (a). The greyscale window is $[-0.1,1.1]$. 


\section{SUMMARY}

In this study, we develop an LRME-STIR method for dynamic PACT image reconstruction. Unlike the conventional FBFIR method, the LRME-STIR reconstruct a sequence of images concurrently. The method exploits both temporal and spatial correlations of the data and thus effectively mitigates image noise. In addition, the method reduces computational burden significantly. When employed in conjunction with advanced iterative image reconstruction algorithm, the LRME-STIR method can reduce data acquisition time, improve temporal resolution, and facilitate four-dimensional PACT applications.

\section{ACKNOWLEDGMENTS}

This work was supported in part by NIH awards EB016963, EB010049, and CA167446. K. W. would also like to thank Dr. Robert W. Schoonover for useful discussions.

\section{REFERENCES}

1. Wang, X., Pang, Y., Ku, G., Xie, X., Stoica, G., and Wang, L. V., "Noninvasive laser-induced photoacoustic tomography for structural and functional in vivo imaging of the brain," Nat. Biotechnol. 21(7), 803-806 (2003).

2. Yang, S., Zhou, Q., Xiang, L., and Lao, Y., "Functional imaging of cerebrovascular activities in small animals using high-resolution photoacoustic tomography," Med. Phys. 34, 3294 (2007).

3. Brecht, H.-P., Su, R., Fronheiser, M., Ermilov, S. A., Conjusteau, A., and Oraevsky, A. A., "Wholebody three-dimensional optoacoustic tomography system for small animals," J. Biomed. Opt. 14(6), 064007 (2009).

4. Wang, L. V., "Tutorial on photoacoustic microscopy and computed tomography," IEEE J. Sel. Top. Quant. 14, 171-179 (2008).

5. Oraevsky, A. A. and Karabutov, A. A., "Optoacoustic tomography," in [Biomedical Photonics Handbook], Vo-Dinh, T., ed., CRC Press LLC (2003).

6. Kruger, R., Reinecke, D., and Kruger, G., "Thermoacoustic computed tomography- technical considerations," Med. Phys. 26, 1832-1837 (1999).

7. Wang, K. and Anastasio, M. A., "Photoacoustic and thermoacoustic tomography: image formation principles," in [Handbook of Mathematical Methods in Imaging], Scherzer, O., ed., Springer (2011).

8. Gamelin, J., Maurudis, A., Aguirre, A., Huang, F., Guo, P., Wang, L. V., and Zhu, Q., "A real-time photoacoustic tomography system for small animals," Opt. Express 17, 10489-10498 (Jun 2009).

9. Li, C., Aguirre, A., Gamelin, J., Maurudis, A., Zhu, Q., and Wang, L. V., "Real-time photoacoustic tomography of cortical hemodynamics in small animals," J. Biomed. Opt. 15(1), 010509-010509-3 (2010).

10. Buehler, A., Herzog, E., Razansky, D., and Ntziachristos, V., "Video rate optoacoustic tomography of mouse kidney perfusion," Opt. Lett. 35, 2475-2477 (Jul 2010).

11. Xiang, L., Wang, B., Ji, L., and Jiang, H., "4-D photoacoustic tomography," Sci. Rep. 3(1113) (2013).

12. Chatni, M. R., Xia, J., Sohn, R., Maslov, K., Guo, Z., Zhang, Y., Wang, K., Xia, Y., Anastasio, M., Arbeit, J., and Wang, L. V., "Tumor glucose metabolism imaged in vivo in small animals with whole-body photoacoustic computed tomography," J. Biomed. Opt. 17(7), 076012-1-076012-7 (2012).

13. Wernick, M., Infusino, E., and Milosevic, M., "Fast spatio-temporal image reconstruction for dynamic PET," IEEE T. Med. Imaging 18(3), 185-195 (1999).

14. Lalush, D. S. and Tsui, B. M. W., "Block-iterative techniques for fast $4 \mathrm{D}$ reconstruction using a priori motion models in gated cardiac SPECT," Phys. Med. Biol. 43(4), 875 (1998).

15. Haldar, J. and Liang, Z.-P., "Spatiotemporal imaging with partially separable functions: A matrix recovery approach," in [Biomedical Imaging: From Nano to Macro, 2010 IEEE International Symposium on], 716719 (2010).

16. Gamelin, J. K., Aguirre, A., and Zhu, Q., "Fast, limited-data photoacoustic imaging for multiplexed systems using a frequency-domain estimation technique," Med. Phys. 38(3), 1503-1518 (2011).

17. Glatz, J., Deliolanis, N. C., Buehler, A., Razansky, D., and Ntziachristos, V., "Blind source unmixing in multi-spectral optoacoustic tomography," Opt. Express 19, 3175-3184 (Feb 2011). 
18. Barrett, H. and Myers, K., [Foundations of Image Science], Wiley Series in Pure and Applied Optics (2004).

19. Wernick, M. N. and Aarsvold, J. N., [Emission Tomography, the Fundamentals of PET and SPECT], Elsevier Academic Press, San Diego, California (2004).

20. Gao, H., Cai, J.-F., Shen, Z., and Zhao, H., "Robust principal component analysis-based four-dimensional computed tomography," Phys. Med. Biol. 56(11), 3181 (2011).

21. Brinegar, C., Schmitter, S. S., Mistry, N. N., Johnson, G. A., and Liang, Z.-P., "Improving temporal resolution of pulmonary perfusion imaging in rats using the partially separable functions model," Magn. Reson. Med. 64(4), 1162-1170 (2010).

22. Qi, W., Yang, Y., Niu, X., and King, M. A., "A quantitative study of motion estimation methods on 4D cardiac gated SPECT reconstruction," Med. Phys. 39(8), 5182-5193 (2012).

23. Wang, L. V. and Wu, H.-I., [Biomedical Optics, Principles and Imaging], Wiley, Hoboken, N.J. (2007).

24. Wang, K., Ermilov, S. A., Su, R., Brecht, H.-P., Oraevsky, A. A., and Anastasio, M. A., "An imaging model incorporating ultrasonic transducer properties for three-dimensional optoacoustic tomography," IEEE T. Med. Imaging 30, $203-214$ (feb. 2011).

25. Wang, K., Su, R., Oraevsky, A. A., and Anastasio, M. A., "Investigation of iterative image reconstruction in three-dimensional optoacoustic tomography," Phys. Med. Biol. 57(17), 5399 (2012).

26. Conjusteau, A., Ermilov, S. A., Su, R., Brecht, H.-P., Fronheiser, M. P., and Oraevsky, A. A., "Measurement of the spectral directivity of optoacoustic and ultrasonic transducers with a laser ultrasonic source," Rev. Sci. Instrum. 80, $093708-093708-5$ (sep 2009).

27. Rosenthal, A., Ntziachristos, V., and Razansky, D., "Model-based optoacoustic inversion with arbitraryshape detectors," Med. Phys. 38(7), 4285-4295 (2011).

28. Finch, D., Patch, S., and Rakesh, "Determining a function from its mean values over a family of spheres," SIAM J. Math. Anal. 35, 1213-1240 (2004).

29. Xu, Y. and Wang, L. V., "Universal back-projection algorithm for photoacoustic computed tomography," Phys. Rev. E 71(016706) (2005).

30. Kunyansky, L. A., "Explicit inversion formulae for the spherical mean Radon transform," Inverse Probl. 23, 373-383 (2007).

31. Kuchment, P. and Kunyansky, L., "Mathematics of photoacoustic and thermoacoustic tomography," in [Handbook of Mathematical Methods in Imaging], Scherzer, O., ed., 817-865, Springer New York (2011).

32. Wang, K., Su, R., Oraevsky, A. A., and Anastasio, M. A., "Sparsity regularized data-space restoration in optoacoustic tomography," Photons Plus Ultrasound: Imaging and Sensing 2012 8223, 822322-822322-7, SPIE (2012).

33. Donoho, D., "De-noising by soft-thresholding," Information Theory, IEEE Transactions on 41(3), 613-627 (1995).

34. Buades, A., Coll, B., and Morel, J., "A review of image denoising algorithms, with a new one," Multiscale Model. Sim. 4(2), 490-530 (2005).

35. Starck, J.-L., Candes, E., and Donoho, D., "The curvelet transform for image denoising," Image Processing, IEEE Transactions on 11, 670-684 (jun 2002).

36. Candès, E. and Recht, B., "Exact matrix completion via convex optimization," Foundations of Computational Mathematics 9(6), 717-772 (2009).

37. Donoho, D. L. and Gavish, M., "The Optimal Hard Threshold for Singular Values is 4/sqrt(3)," ArXiv e-prints (May 2013).

38. Candes, E. J., Sing-Long, C. A., and Trzasko, J. D., "Unbiased Risk Estimates for Singular Value Thresholding and Spectral Estimators," ArXiv e-prints (Oct. 2012).

39. Xia, J., Chatni, M. R., Maslov, K., Guo, Z., Wang, K., Anastasio, M., and Wang, L. V., "Whole-body ring-shaped confocal photoacoustic computed tomography of small animals in vivo," J. Biomed. Opt. 17(5), 050506-1-050506-3 (2012).

40. Finch, D., Haltmeier, M., and Rakesh, "Inversion of spherical means and the wave equation in even dimensions," SIAM Journal on Applied Mathematics 68(2), 392-412 (2007). 